\title{
Production of inflammatory cytokines, cortisol, and $A \beta I-40$ in elderly oral cancer patients with postoperative delirium
}

This article was published in the following Dove Press journal:

Neuropsychiatric Disease and Treatment

27 October 2016

Number of times this article has been viewed

\author{
Lulu Sun ${ }^{1, *}$ \\ Peiyu Jia ${ }^{2}$ \\ Junfeng Zhang ${ }^{2}$ \\ Xin Zhang ${ }^{2}$ \\ Yiwei Zhang ${ }^{2}$ \\ Hong Jiang' \\ Wei Jiang ${ }^{2}$ \\ Yong Guo 2,* \\ 'Department of Anesthesiology and \\ Critical Care Medicine, Shanghai \\ Ninth People's Hospital Affiliated \\ to Shanghai Jiao Tong University \\ School of Medicine, ${ }^{2}$ Department \\ of Anesthesiology and Critical \\ Care Medicine, Shanghai Jiao Tong \\ University Affiliated Sixth People's \\ Hospital, Shanghai, People's Republic \\ of China \\ *These authors contributed equally \\ to this work
}

Aim: Pathophysiological disorders after surgery might be related to postoperative delirium (POD). This study was designed to elucidate the pathogenesis of POD in elderly oral cancer patients by determining the perioperative kinetics of inflammatory cytokines, cortisol, and amyloid $\beta 1-40$ (A $\beta 1-40)$.

Methods: A total of 257 elderly oral cancer patients who underwent tumor resection surgery were selected. Venous blood was collected prior to surgery (T0), at the end of surgery (T1), and at 12 hours after surgery (T2). During the first three postoperative days, patients were examined using the confusion assessment method twice a day ( 8 am and $8 \mathrm{pm}$ ). Mini-Mental State Examination scores were recorded at $\mathrm{T} 0$ and on postoperative days 1, 3, and 7. Ultimately, 56 patients suffering from POD made up the POD group, and 56 patients randomly selected from a cohort of patients without POD were allocated to the no POD (NPOD) group. Subsequently, interleukin-6, C-reactive protein, procalcitonin, cortisol, and $\mathrm{A} \beta 1-40$ in plasma from the two groups were measured.

Results: The two groups displayed comparable basic characteristics. There were no differences in all tested biomarkers between the two groups at T0. However, after surgery, the biomarker levels displayed distinct patterns between the two groups. The peak levels of all biomarkers were higher in the POD group than in the NPOD group. Conversely, the Mini-Mental State Examination scores after surgery were lower in the POD group than in the no POD group.

Conclusion: The boost of inflammatory cytokines, cortisol, and A $\beta 1-40$ after surgery might be involved in POD onset among elderly oral cancer patients. POD was accompanied by progressive cognitive deficiency.

Keywords: elderly, postoperative delirium, inflammatory cytokines, cortisol, $\beta$-amyloid protein

\section{Introduction}

Acute attack, illness fluctuation, attention deficit, confusion, and disturbance of consciousness are core features of postoperative delirium (POD). ${ }^{1}$ Elderly patients with preoperative cognitive impairment, dementia, anxiety, and depression are more prone to POD. ${ }^{2}$ Elderly oral cancer patients who undergo tumor resection combined with free flap surgery experience a prolonged operation time, severe trauma, long-term artificial airway placement, and postoperative restraint due to the need for a flap. These predisposing factors lead to a high incidence of POD in elderly oral cancer patients. . $^{3,4}$ The negative consequences of POD include an increased burden of care, a prolonged hospital stay, an elevated rate of mortality during hospitalization, and long-term cognitive impairment or even permanent dementia. ${ }^{5}$ Excessive inflammatory responses
Correspondence: Yong Guo; Wei Jiang Department of Anesthesiology and Critical Care Medicine, Shanghai Sixth People's Hospital, 600 Yishan

Road, Shanghai 200233, People's

Republic of China

Tel +862l 64369181

Email garfield.guo@sjtu.edu.cn;

dr_jiangwei@163.com 
induced by surgery may lead to impaired mental status postoperatively. ${ }^{6}$ However, the pathophysiological mechanisms underlying POD remain to be elucidated. Recent emerging hypotheses include "neuroinflammatory", "neuronal aging", and "neuroendocrine". ${ }^{7}$ This study was designed to elucidate the pathogenesis of POD by observing the kinetics of inflammation, stress, and dementia markers in elderly oral cancer patients with POD.

\section{Methods}

\section{Patients and setting}

A total of 257 elderly oral cancer patients who underwent tumor resection together with free flap surgery under general anesthesia in the past several months were selected. The inclusion criteria were as follows: American Society of Anesthesiologists physical status I-III; age $\geq 65$ years and $\leq 80$ years; and duration of postoperative stay in the surgical intensive care unit $\geq 3$ days. The exclusion criteria were Mini-Mental State Examination (MMSE) score $<24$ or dementia of various etiologies; history of central nervous system (CNS) or mental illness; current use of sedatives or antidepressants; history of neurosurgery; history of endocrine and metabolic disorders; history of inflammatory disease; recent use of glucocorticoids, cytokines, or anti-cytokine therapy; complications of secondary surgery or severe infections (we used the presence of more than two systemic inflammatory response syndrome criteria as a marker of inflammation) ${ }^{8}$ unwilling to complete the experimental procedures or language barriers to participation; severe hearing or visual impairment; illiteracy; and alcohol or drug dependence. This study was approved by the Ethics Committee of Shanghai Ninth People's Hospital affiliated to Shanghai Jiao Tong University School of Medicine (Shanghai, People's Republic of China), and written informed consent was provided by all patients.

Before surgery, the patients were interviewed and assessed. The demographic data and the patients' medical histories were recorded and MMSE and Charlson's Comorbidity Index were conducted.

\section{Anesthesia, surgery, and postoperative pain treatment}

No patients received medication prior to surgery. Systolic blood pressure, diastolic blood pressure, pulse oxygen saturation, electrocardiogram, stroke volume variation, bispectral index, and end-tidal $\mathrm{CO}_{2}$ were continuously monitored during the perioperative period. Anesthesia was induced with midazolam, sufentanil, propofol, and vecuronium. Anesthesia was maintained via inhalation of $1 \%-3 \%$ isoflurane, continuous intravenous infusion of propofol, and intermittent infusion of sufentanil and vecuronium to maintain analgesic and muscle relaxant effects. During surgery, the respiratory parameters of the anesthesia machine were modulated to maintain an end-tidal $\mathrm{CO}_{2}$ between 30 and $40 \mathrm{mmHg}$, and the depth of anesthesia was adjusted to maintain the bispectral index value within the range of 40-60. Intraoperative blood pressure fluctuations were maintained within $\pm 30 \%$ of the baseline values, and the heart rate was sustained at 50-100 beats/min. All patients underwent tracheotomy after the operation, followed by transport to the surgical intensive care unit. After surgery, all patients were continuously administered analgesia via a pump in the same manner. Moreover, identical treatments, such as anti-infection practices, phlegm elimination, and detumescence, were provided to all patients. All included patients did not receive sedatives after surgery.

\section{Blood sample collection and sample bank establishment}

Venous blood was drawn through a femoral vein catheter prior to surgery (T0), at the end of surgery (T1), and 12 hours after surgery (T2). Blood samples were stored in anticoagulant tubes and immediately centrifuged at $4^{\circ} \mathrm{C}$ for 10 minutes $(3,000 \mathrm{rpm})$. Then, the plasma samples were frozen in $\mathrm{a}-80^{\circ} \mathrm{C}$ refrigerator (Sanyo, Osaka, Japan) until analysis.

\section{POD assessment and group allocation}

During the first 3 days after surgery, patients were assessed by an expert in psychology using the confusion assessment method twice a day ( 8 am and $8 \mathrm{pm}$ ). The confusion assessment method was used to assess delirium; this instrument contains four items: 1) acute change or fluctuating course of mental status; 2) inattention; 3 ) altered level of consciousness; and 4) disorganized thinking. A positive delirium diagnosis requires the presence of items 1) and 2) as well as either item 3) or 4). ${ }^{9}$

The confusion assessment method is a simple and rapid method for evaluating the state of consciousness according to the Diagnostic and Statistical Manual of Mental Disorders Fourth Edition.

In total, 257 patients were included in the study. Among this cohort, 56 patients exhibited POD and were allocated to the POD group. An additional 56 patients were randomly selected from the cohort of patients without POD and were allocated to the no POD (NPOD) group. Moreover, the MMSE scores of the two groups were recorded at T0 and on postoperative days 1,3 , and 7 by the same doctor in the morning. 


\section{Blood tests}

The levels of interleukin (IL)-6, C-reactive protein (CRP), procalcitonin (PCT), cortisol, and amyloid $\beta 1-40$ (A $\beta 1-40)$ in plasma from the two groups were determined. The IL-6 levels were measured via enzyme-linked immunosorbent assay (R\&D Systems, Inc., Minneapolis, MN, USA). PCT was measured via an immunometric assay using time-resolved amplified cryptate emission technology (PCT-sensitive Kryptor kit; Brahms, Middletown, VA, USA). Analyses of the CRP and cortisol levels were performed using the VITROS 5600 analyzer (Johnson and Johnson, New Brunswick, NJ, USA). The A $1-40$ concentrations were measured via enzyme-linked immunosorbent assay (Sigma-Aldrich Co., St Louis, MO, USA). Enzyme-linked immunosorbent assay was carried out with a standard protocol and adopted chromogenic reporters in a quantitative format to measure $\beta$-A $\beta$ specific activity. We employed the Shanghai Zhili Biological Technology Company (Shanghai, People's Republic of China) to build up reaction standard curves. The protein level was determined while comparing the optical density value of samples and the standard curve. All procedures were performed according to the manufacturer's instructions.

\section{Statistical analysis}

Descriptive results of continuous variables are expressed as mean \pm standard deviation. Categorical data are expressed as counts. The Kolmogorov-Smirnov test was used to test for normality. If continuous variables were not normally distributed, the Mann-Whitney $U$-test was used. Otherwise, Student's $t$-test was applied (eg, age, education, operation duration, anesthesia duration, volume of infusion). Quantitative data (eg, IL-6, CRP, PCT, cortisol, A $\beta 1-40$ ) between groups were analyzed by the Student's $t$-test and intragroup numerical data were analyzed by repeated measures analysis of variance. Enumeration data (eg, female, American Society of Anesthesiologists grade, flap reconstruction) were analyzed by chi-squared test. A $P$-value $<0.05$ was considered significant. SPSS 16.0 (SPSS Inc., Chicago, IL, USA) was used for statistical analysis.

\section{Results}

Demographic data for the patients are shown in Table 1. The incidence of POD in elderly oral cancer patients undergoing tumor resection combined with free flap surgery was $21.8 \%$ (56/257). There were no significant differences in age, sex, weight, education level, American Society of Anesthesiologists grade, or other basic characteristics between the POD and NPOD groups $(P>0.05)$.
Table I Demographic characteristics of the POD and NPOD groups

\begin{tabular}{llll}
\hline Characteristic & POD $(\mathbf{n}=\mathbf{5 6})$ & NPOD $(\mathbf{n}=\mathbf{5 6})$ & P-value \\
\hline Age (years) & $73.2 \pm 6.5$ & $72.7 \pm 8.3$ & 0.72 \\
Female & 29 & 33 & 0.57 \\
Weight (kg) & $60 \pm 15$ & $63 \pm 16$ & 0.31 \\
Education (years) & $8.2 \pm 2.3$ & $8.5 \pm 2.8$ & 0.54 \\
ASA grade (I/II/III) & $9 / 41 / 5$ & $15 / 36 / 6$ & 0.40 \\
MMSE score at T0 & $27.91 \pm 1.53$ & $28.13 \pm 1.62$ & 0.46 \\
Charlson's Comorbidity & $1.5 \pm 0.7$ & $1.7 \pm 0.8$ & 0.16 \\
Index at T0 & & & \\
Flap reconstruction & & & 0.36 \\
$\quad$ Forearm & 25 & 24 & \\
$\quad$ Anterolateral thigh & 20 & 15 & \\
$\quad$ Peroneal muscles & 11 & 17 & \\
Operation duration (min) & $481 \pm 102$ & $470 \pm 108$ & 0.58 \\
Anesthesia duration (min) & $515 \pm 97$ & $506 \pm 104$ & 0.64 \\
Blood loss (mL) & $802 \pm 211$ & $785 \pm 203$ & 0.66 \\
RBC transfusion (units) & $2.6 \pm 1.8$ & $2.1 \pm 1.9$ & 0.16 \\
FFP transfusion (units) & $2.2 \pm 1.6$ & $1.8 \pm 1.5$ & 0.18 \\
Volume of infusion (mL) & $3,612.0 \pm 613.9$ & $3,498.5 \pm 656.5$ & 0.35 \\
\hline
\end{tabular}

Note: Data are shown as mean \pm SD or numbers.

Abbreviations: ASA, American Society of Anesthesiologists; FFP, fresh frozen plasma; MMSE, Mini-Mental State Examination; NPOD, no POD; POD, postoperative delirium; RBC, red blood cells; SD, standard deviation; min, minutes; T0, prior to surgery.

A total of 257 patients were included in this study and completed all observations. Among these, 56 patients exhibited POD. Specifically, 31 POD cases began on postoperative day 1,19 on postoperative day 2 , and six on postoperative day 3. As shown in Figure 1, the added morbidity of POD on postoperative day 3 was lower than that on postoperative days $1(P<0.01)$ and $2(P=0.01)$. As shown in Figure 2, among the 56 patients with POD, 38 patients were diagnosed with hyperactive delirium, seven with hypoactive delirium, and eleven with mixed delirium.

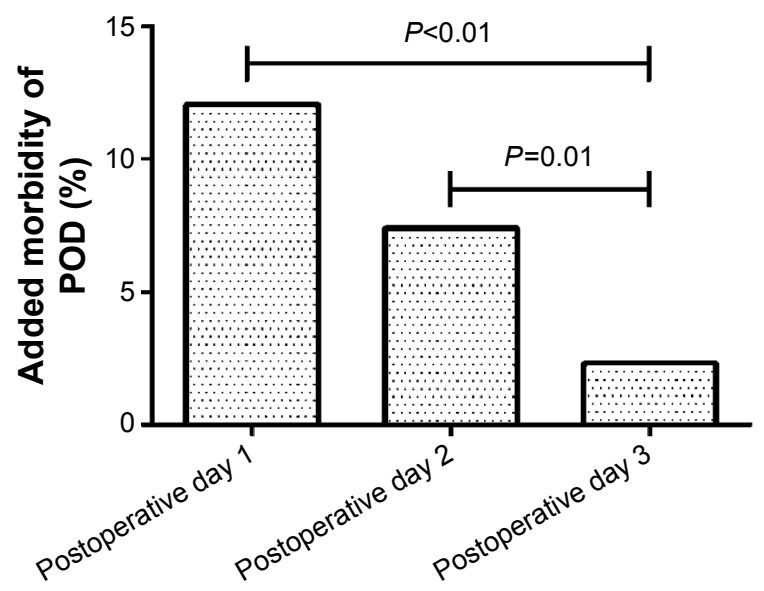

Figure I The added morbidity of postoperative delirium (POD) days I-3. Note: The added morbidity of POD on postoperative day 3 was lower than that on postoperative days I $(P<0.0 \mathrm{I})$ and $2(P=0.0 \mathrm{I})$. 


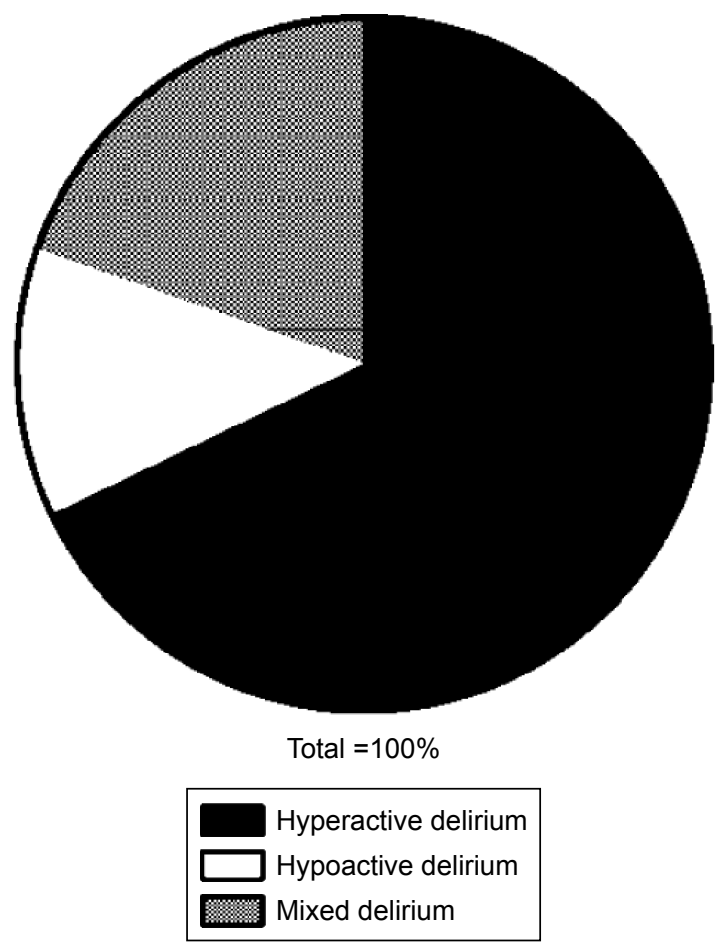

Figure 2 The type of postoperative delirium (POD).

Notes: Among the 56 patients with POD, 38 patients were diagnosed with hyperactive delirium, seven with hypoactive delirium, and eleven with mixed delirium.

As shown in Figure 3, there were no significant differences in the IL-6 levels between the two groups before surgery (T0) $(P=0.60)$. At the end of surgery (T1) and 12 hours after surgery (T2), the IL-6 levels were higher in the POD than that in NPOD group $(158.7 \pm 9.5$ vs $97.9 \pm 10.3 \mathrm{pg} / \mathrm{L}$ at T1, 77.9 \pm 5.6 vs $57.1 \pm 4.6 \mathrm{pg} / \mathrm{L}$ at T2, $P<0.01)$. At T1 and T2, the levels of IL-6 in each group were higher than those in the corresponding group at T0 $(P<0.05)$.

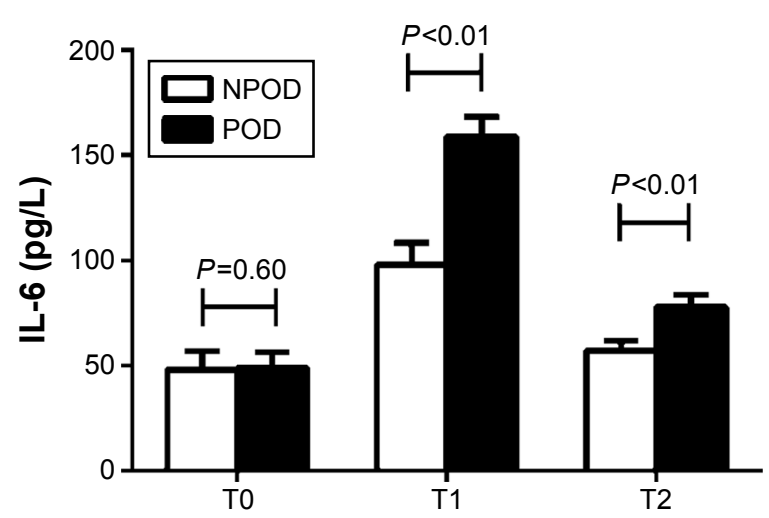

Figure 3 Concentration of interleukin (IL)- 6 between the NPOD and POD groups. Notes: There were no significant differences in the IL-6 levels between the two groups prior to surgery (T0) $(P=0.60)$. At the end of surgery $(\mathrm{TI})$ and $12 \mathrm{~h}$ after surgery (T2), the IL-6 levels were higher in the POD than that in NPOD group $(P<0.0 \mathrm{I})$. At $\mathrm{TI}$ and $\mathrm{T} 2$, the levels of IL-6 in each group were higher than those in the corresponding group at T0 $(P<0.05)$.

Abbreviations: NPOD, no POD; POD, postoperative delirium; h, hours.

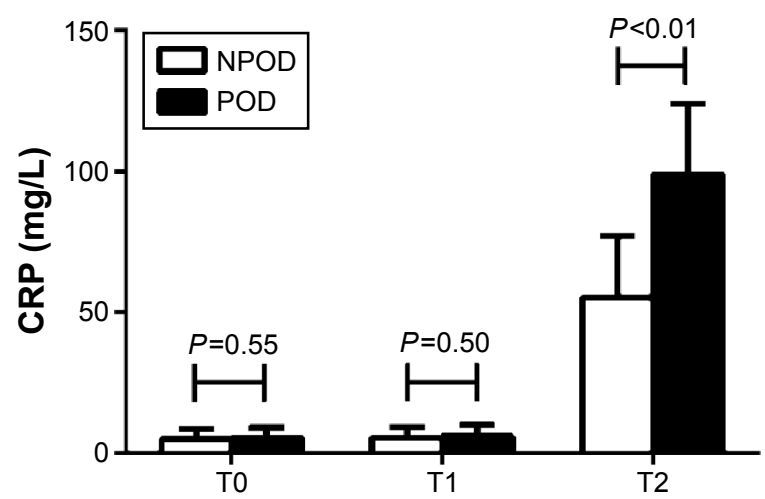

Figure 4 Concentration of CRP between the NPOD and POD groups.

Notes: There were no significant differences in the CRP levels between the two groups prior to surgery (T0) $(P=0.55)$ and at the end of surgery $(\mathrm{TI})(P=0.50)$. Furthermore, the CRP levels in each group at TI were not different from those in the corresponding group at T0 $(P>0.05)$. However, $12 \mathrm{~h}$ after surgery (T2), the CRP levels were higher in the POD than that in NPOD group $(P<0.01)$. At $T 2$, the levels of CRP in each group were higher than those in the corresponding group at T0 $(P<0.05)$.

Abbreviations: CRP, C-reactive protein; NPOD, no POD; POD, postoperative delirium; h, hours.

As shown in Figure 4, there were no significant differences in the CRP levels between the two groups before surgery (T0) $(P=0.55)$ and at the end of surgery (T1) $(P=0.50)$. Furthermore, the CRP levels in each group at T1 were not different from those in the corresponding group at T0 $(P>0.05)$. However, 12 hours after surgery (T2), the CRP levels were higher in the POD than that in NPOD group $(98.8 \pm 25.3$ vs $55.2 \pm 21.8 \mathrm{mg} / \mathrm{L}, P<0.01)$. At T2, the levels of CRP in each group were higher than those in the corresponding group at T0 $(P<0.05)$.

As shown in Figure 5, there were no significant differences in the PCT levels between the two groups before surgery (T0) $(P=0.14)$. At the end of surgery (T1) and 12 hours after surgery (T2), the PCT levels were higher in the POD

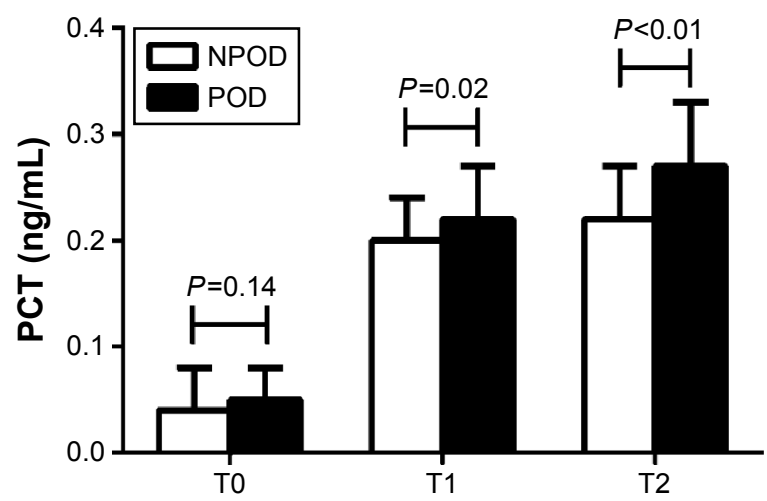

Figure 5 Concentration of PCT between the NPOD and POD groups.

Notes: There were no significant differences in the PCT levels between the two groups prior to surgery (TO) $(P=0.14)$. At the end of surgery $(\mathrm{TI})$ and $12 \mathrm{~h}$ after surgery (T2), the PCT levels were higher in the POD than that in NPOD group $(P=0.02$ and $P<0.01)$. At $T I$ and $T 2$, the levels of PCT in each group were higher than those in the corresponding group at T0 $(P<0.05)$.

Abbreviations: NPOD, no POD; POD, postoperative delirium; PCT, procalcitonin; h, hours. 


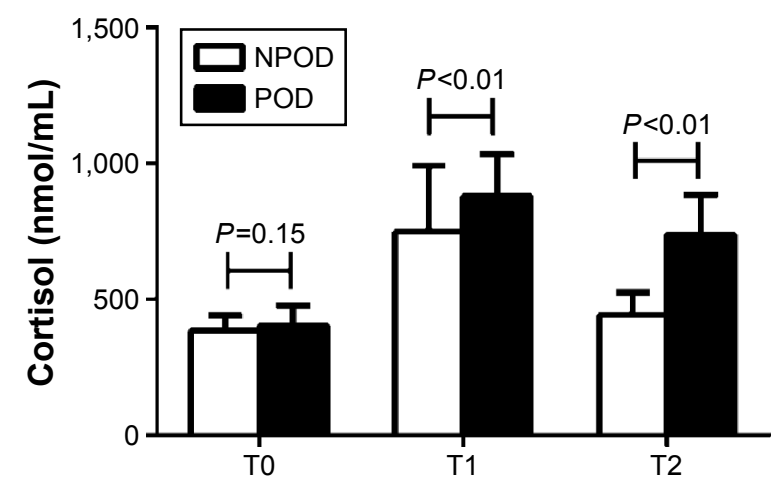

Figure 6 Concentration of cortisol between the NPOD and POD groups. Notes: There were no significant differences in the cortisol levels between the two groups prior to surgery (T0) $(P=0.15)$. At the end of surgery $(\mathrm{TI})$ and $12 \mathrm{~h}$ after surgery (T2), the cortisol levels were higher in the POD than that in NPOD group $(P<0.0 \mathrm{I})$. At $\mathrm{TI}$ and $\mathrm{T} 2$, the levels of cortisol in each group were higher than those in the corresponding group at T0 $(P<0.05)$.

Abbreviations: NPOD, no POD; POD, postoperative delirium; h, hours

than that in NPOD group $(0.22 \pm 0.05$ vs $0.20 \pm 0.04 \mathrm{ng} / \mathrm{mL}$ at $\mathrm{T} 1, P=0.02 ; 0.27 \pm 0.06$ vs $0.22 \pm 0.05 \mathrm{ng} / \mathrm{mL}$ at T2, $P<0.01$ ). At T1 and T2, the levels of PCT in each group were higher than those in the corresponding group at T0 $(P<0.05)$.

As shown in Figure 6, there were no significant differences in the cortisol levels between the two groups before surgery (T0) $(P=0.15)$. At the end of surgery (T1) and 12 hours after surgery (T2), the cortisol levels were higher in the POD than that in NPOD group $(878.9 \pm 153.8 \mathrm{vs} 749.1 \pm 241.9 \mathrm{nmmol} / \mathrm{mL}$ at T1, 736.8 \pm 147.8 vs $441.9 \pm 82.2 \mathrm{nmmol} / \mathrm{mL}$ at T2, $P<0.01)$. At T1 and T2, the levels of cortisol in each group were higher than those in the corresponding group at T0 $(P<0.05)$.

As shown in Figure 7, there were no significant differences in the $A \beta 1-40$ levels between the two groups

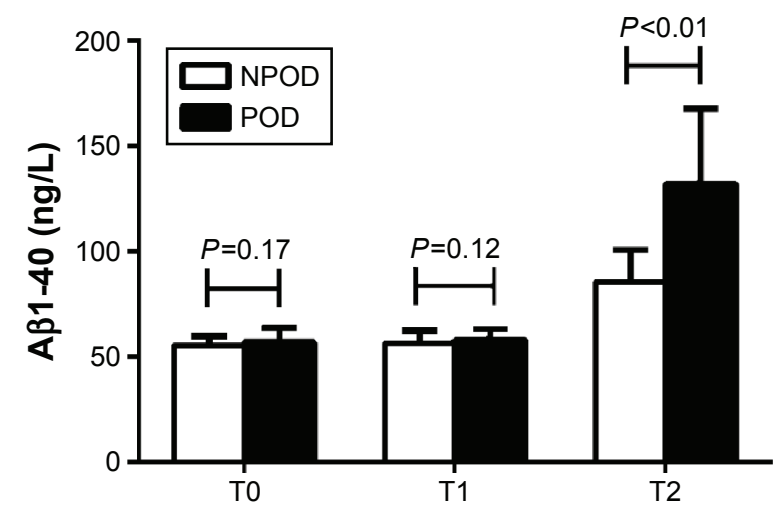

Figure 7 Concentration of $A \beta I-40$ between the NPOD and POD groups. Notes: There were no significant differences in the $A \beta \mid-40$ levels between the two groups prior to surgery (T0) $(P=0.17)$ and at the end of surgery $(T I)(P=0.12)$. Furthermore, the $A \beta I-40$ levels in each group at TI were not different from those in the corresponding group at T0 $(P>0.05)$. However, $12 \mathrm{~h}$ after surgery (T2), the $A \beta I-40$ levels were higher in the POD than that in NPOD group $(P<0.01)$. At T2, the levels of $A \beta \mathrm{I}-40$ in each group were higher than those in the corresponding group at T0 $(P<0.05)$.

Abbreviations: $A \beta \mid-40$, amyloid $\beta \mathrm{I}-40$; NPOD, no POD; POD, postoperative delirium; h, hours.

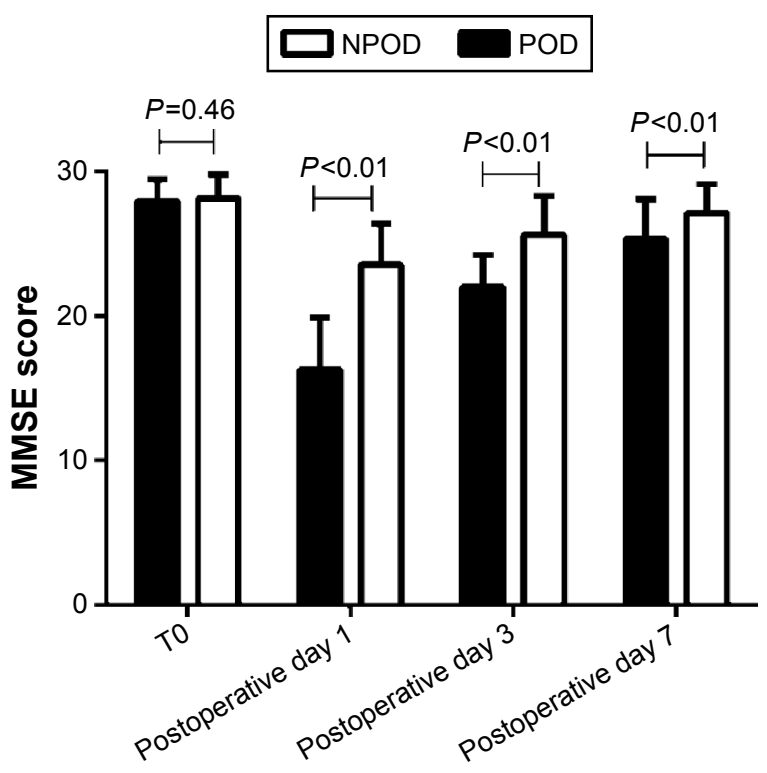

Figure 8 MMSE scores of the NPOD and POD groups during the perioperative period.

Note: No differences in the MMSE scores were found between the two groups at TO $(P=0.46)$, but the MMSE scores after surgery were lower in the POD than that in NPOD group $(P<0.01)$.

Abbreviations: MMSE, Mini-Mental State Examination; NPOD, no POD; POD, postoperative delirium; T0, prior to surgery.

before surgery (T0) $(P=0.17)$ and at the end of surgery (T1) $(P=0.12)$. Furthermore, the A $\beta 1-40$ levels in each group at T1 were not different from those in the corresponding group at T0 $(P>0.05)$. However, 12 hours after surgery (T2), the $A \beta 1-40$ levels were higher in the POD than that in NPOD group (132.1 \pm 35.7 vs $85.2 \pm 15.6 \mathrm{ng} / \mathrm{L}, P<0.01)$. At T2, the levels of $A \beta 1-40$ in each group were higher than those in the corresponding group at T0 $(P<0.05)$.

As shown in Figure 8, no difference in the MMSE score at T0 was found between the two groups $(P=0.46)$, but the MMSE scores after surgery were lower in the POD than in NPOD group (16.25 \pm 3.63 vs $23.53 \pm 2.85$ on postoperative day $1,22.01 \pm 2.19$ vs $25.61 \pm 2.67$ on postoperative day $2,25.31 \pm 2.75$ vs $27.11 \pm 2.01$ on postoperative day $3, P<0.01)$.

\section{Discussion}

In our study, the incidence of POD among elderly oral cancer patients was $20.7 \%$. This value was similar to the result reported by Shiiba et al. ${ }^{10}$ Besides, POD primarily occurred during the period of 24-72 hours after surgery, and most cases were hyperactivity delirium.

The results of this study also suggested that there were no baseline differences in the levels of IL-6, CRP, PCT, cortisol, and $A \beta 1-40$ between the two groups. However, the levels of IL-6, PCT, and cortisol appeared to increase during the early postoperative period, whereas the CRP 
and $A \beta 1-40$ levels began to rise later. The peak levels of all tested markers were higher in the POD than that in NPOD group. Furthermore, the POD group exhibited more intense inflammation and stress responses than NPOD group. Surgical trauma can lead to activation of the immune system and hyperactivity of the hypothalamic-pituitary-adrenal axis, leading to aberrancies in the levels of acetylcholine, norepinephrine, 5-hydroxytryptamine, and other neurotransmitters in the CNS. These aberrancies might ultimately result in brain dysfunction after surgery. Beloosesky et al found that the serum levels of IL-6, IL-8, IL-10, and CRP in elderly hip fracture patients drastically increased early after fracture and then decreased up to 30 days after surgery, and these changes were related to impaired mental status. ${ }^{6}$

Peripheral inflammatory cytokines released as a result of surgery could enter the CNS through transporters to cross the blood-brain barrier; these cytokines could subsequently activate microglia to produce an inflammatory response. Inflammatory cytokines could interfere with the connection and transmission functions of synapses. Intracerebroventricular injection of IL-6 not only caused a significant inflammatory response by glial cells but also led to reduced dendritic arborization, decreased cholinergic innervation in the hippocampus, enhanced long-term potentiation of synaptic transmission, increased complement protein synthesis, hampered behavioral performance, and significantly impaired learning and memory functions. ${ }^{11}$ This study found that the peak levels of IL-6 were higher in the POD than in NPOD group. This observation indicates that there was more intense and prolonged CNS inflammation, which could lead to neurological dysfunction, in the POD compared to NPOD group.

Neuroendocrine cells or macrophages in the liver, lung, spleen, and kidney can generate PCT upon stimulation by bacterial endotoxins, tumor necrosis factor- $\alpha$, IL- 6 , IL-2, or other factors. Tumor necrosis factor- $\alpha$ and IL- 6 regulate PCT production. CRP appears in serum during the acute phase of infection, tissue injury, ischemia, malignant tumor growth, and other disorders; therefore, this marker has poor specificity. Elevated CRP levels are associated with the prevalence of dementia among the very old. ${ }^{12}$ The dynamics of IL-6, CRP, and PCT levels observed in this study were analogous to an inflammatory response. After healthy volunteers received an injection of endotoxin, the CRP levels exceeded the normal values 8-12 hours later and remained high at 24-72 hours after injection; the PCT concentrations began to rise after 4 hours and were sustained at a high level at $8-24$ hours after injection; and the tumor necrosis factor- $\alpha$ and IL-6 levels peaked at 2-3 hours but became undetectable beyond 24 hours after injection. ${ }^{13}$ Our study found that the PCT concentrations were $\sim 0.1 \mathrm{ng} / \mathrm{mL}$ before surgery and gradually increased after surgery, peaking at $\sim 0.5 \mathrm{ng} / \mathrm{mL}$. This value reflected only localized bacterial infection after surgery. ${ }^{14}$ The peak levels of PCT and CRP were higher in the POD than in NPOD group, which indicated more intense and persistent inflammation in the POD group.

$A \beta 1-40$ and $A \beta 1-42$ are major forms of $\beta$-amyloid protein. When the generation or aggregation of $\beta$-amyloid protein increases excessively, amyloid plaques are formed and deposited between neurons. This event causes neuronal loss, which is an important pathophysiological process in the pathogenesis of Alzheimer's disease (AD). At present, several studies suggest that surgery under general anesthesia is associated with $\mathrm{AD}$ or POD. ${ }^{15,16} \mathrm{Xie}$ et al found that a clinically relevant concentration of isoflurane induced neuronal apoptosis and increased $\beta$-amyloid protein production in a human neuroglioma cell line. ${ }^{17,18}$ Moreover, the plasma A $\beta 1-40$ levels showed a significant association with poor neuropsychological performance in the aged. ${ }^{19}$ Our study revealed that the $A \beta 1-40$ concentrations were increased 12 hours after surgery and that the $A \beta 1-40$ levels were higher in the POD than in NPOD group. The boost of A $\beta 1-40$ after surgery might facilitate the development of POD.

Postoperative neurological disorders include POD and postoperative cognitive dysfunction. POD is generally believed to have an acute onset within 3 days after surgery. Some patients with POD recover their impaired cognitive function during hospitalization, but others continue to experience impaired cognitive performance for an extended period after surgery. ${ }^{20-22}$ Nevertheless, postoperative cognitive dysfunction typically occurs during the period of 3-7 days after surgery. Our results suggested that those elderly oral cancer patients with POD would suffer more serious and prolonged cognitive deficiency.

\section{Conclusion}

In summary, the elevation of the levels of inflammatory cytokines, cortisol, and A $\beta 1-40$ after surgery under general anesthesia might be involved in the onset of POD among elderly oral cancer patients. Early POD might subsequently induce more severe cognitive deficiency. These results may have implications for the prevention and treatment of POD.

\section{Acknowledgments}

This work was supported by Shanghai Municipal Commission of Health and Family Planning Foundation for Key 
Developing Disciplines (2015ZB0103) and Shanghai Jiao Tong University Biomedical Engineering Cross Research Foundation (YG2015MS15). The content is solely the responsibility of the authors.

\section{Author contributions}

All authors contributed toward data analysis, drafting, and revising the paper and agree to be accountable for all aspects of the work.

\section{Disclosure}

The authors report no conflicts of interest in this work.

\section{References}

1. Inouye SK, Schlesinger MJ, Lydon TJ. Delirium: a symptom of how hospital care is failing older persons and a window to improve quality of hospital care. Am J Med. 1999;106(5):565-573.

2. de Castro SM, Unlu C, Tuynman JB, et al. Incidence and risk factors of delirium in the elderly general surgical patient. Am J Surg. 2014;208(1): 26-32.

3. Yang R, Lubek JE, Dyalram D, Liu X, Ord RA. Head and neck cancer surgery in an elderly patient population: a retrospective review. Int $J$ Oral Maxillofac Surg. 2014;43(12):1413-1417.

4. Takeuchi M, Takeuchi H, Fujisawa D, et al. Incidence and risk factors of postoperative delirium in patients with esophageal cancer. Ann Surg Oncol. 2012;19(12):3963-3970.

5. Skrobik Y. Delirium prevention and treatment. Anesthesiol Clin. 2011; 29(4):721-727.

6. Beloosesky Y, Hendel D, Weiss A, et al. Cytokines and C-reactive protein production in hip-fracture-operated elderly patients. $J$ Gerontol A Biol Sci Med Sci. 2007;62(4):420-426.

7. Maldonado JR. Neuropathogenesis of delirium: review of current etiologic theories and common pathways. Am J Geriatr Psychiatry. 2013;21(12):1190-1222.

8. American College of Chest Physicians/Society of Critical Care Medicine Consensus Conference: definitions for sepsis and organ failure and guidelines for the use of innovative therapies in sepsis. Crit Care Med. 1992;20(6):864-874.
9. Ely EW, Margolin R, Francis J, et al. Evaluation of delirium in critically ill patients: validation of the Confusion Assessment Method for the Intensive Care Unit (CAM-ICU). Crit Care Med. 2001;29(7): 1370-1379.

10. Shiiba M, Takei M, Nakatsuru M, et al. Clinical observations of postoperative delirium after surgery for oral carcinoma. Int J Oral Maxillofac Surg. 2009;38(6):661-665.

11. Campbell IL. Transgenic mice and cytokine actions in the brain: bridging the gap between structural and functional neuropathology. Brain Res Brain Res Rev. 1998;26(2-3):327-336.

12. Kravitz BA, Corrada MM, Kawas CH. Elevated C-reactive protein levels are associated with prevalent dementia in the oldest-old. Alzheimers Dement. 2009;5(4):318-323.

13. Reinhart K, Meisner M, Brunkhorst FM. Markers for sepsis diagnosis: what is useful? Crit Care Clin. 2006;22(3):503-519.

14. Meisner M, Brunkhorst FM, Reith HB, Schmidt J, Lestin HG, Reinhart K. Clinical experiences with a new semi-quantitative solid phase immunoassay for rapid measurement of procalcitonin. Clin Chem Lab Med. 2000;38(10):989-995.

15. Hussain M, Berger M, Eckenhoff RG, Seitz DP. General anesthetic and the risk of dementia in elderly patients: current insights. Clin Interv Aging. 2014;9:1619-1628.

16. Evered LA, Silbert BS, Scott DA, et al. Plasma amyloid beta42 and amyloid beta40 levels are associated with early cognitive dysfunction after cardiac surgery. Ann Thorac Surg. 2009;88(5); $1426-1432$.

17. Xie Z, Dong Y, Maeda U, et al. The common inhalation anesthetic isoflurane induces apoptosis and increases amyloid beta protein levels. Anesthesiology. 2006;104(5):988-994.

18. Xie Z, Dong Y, Maeda U, et al. Isoflurane-induced apoptosis: a potential pathogenic link between delirium and dementia. J Gerontol A Biol Sci Med Sci. 2006;61(12):1300-1306.

19. Rembach A, Faux NG, Watt AD, et al. Changes in plasma amyloid beta in a longitudinal study of aging and Alzheimer's disease. Alzheimers Dement. 2014;10(1):53-61.

20. Wacker P, Nunes PV, Cabrita H, Forlenza OV. Post-operative delirium is associated with poor cognitive outcome and dementia. Dement Geriatr Cogn Disord. 2006;21(4):221-227.

21. Saczynski JS, Marcantonio ER, Quach L, et al. Cognitive trajectories after postoperative delirium. $N$ Engl J Med. 2012;367(1):30-39.

22. McCusker J, Cole M, Dendukuri N, Belzile E, Primeau F. Delirium in older medical inpatients and subsequent cognitive and functional status: a prospective study. CMAJ. 2001;165(5):575-583.
Neuropsychiatric Disease and Treatment

\section{Publish your work in this journal}

Neuropsychiatric Disease and Treatment is an international, peerreviewed journal of clinical therapeutics and pharmacology focusing on concise rapid reporting of clinical or pre-clinical studies on a range of neuropsychiatric and neurological disorders. This journal is indexed on PubMed Central, the 'PsycINFO' database and CAS,

\section{Dovepress}

and is the official journal of The International Neuropsychiatric Association (INA). The manuscript management system is completely online and includes a very quick and fair peer-review system, which is all easy to use. Visit http://www.dovepress.com/testimonials.php to read real quotes from published authors. 